\title{
Physical activity in adults with and without diabetes: from the 'high-risk' approach to the 'population-based' approach of prevention
}

Abla Mehio Sibai ${ }^{1,6^{*}}$, Christy Costanian ${ }^{1}$, Rania Tohme ${ }^{2,3}$, Shafika Assaad ${ }^{4}$ and Nahla Hwalla ${ }^{5,6}$

\begin{abstract}
Background: The prevalence rates of physical inactivity and diabetes in the MENA region are among the highest in the world. However, studies that focus on factors that influence the pattern of physical activity in the region remain very scarce. This study aimed to determine the prevalence and correlates of physical activity in the general population and among subjects with and without diabetes in Lebanon, a small middle-income country in the MENA region.

Methods: We conducted a cross-sectional nation-wide study of 2,195 randomly selected adults aged 25 years and older. Participants completed a comprehensive questionnaire based on the WHO-STEPwise guidelines. Physical activity was assessed using the International Physical Activity Questionnaire. Type 2 diabetes risk factors examined were age $\geq 45$ years, BMl $\geq 25 \mathrm{~kg} / \mathrm{m}^{2}$, hypertension, hyperlipidemia, cardiovascular disease and family history of diabetes.

Results: Close to $10 \%$ of adults with diabetes were physically active versus $23.4 \%$ without diabetes. Prevalence rates of physical activity declined consistently as the number of diabetes risk factors increased. Odds ratios for physical activity were lower among the educated $(0.75,95 \% \mathrm{Cl}=0.57-0.98)$, those who owned at least one car $(0.71,95 \%$ $\mathrm{Cl}=0.57-0.88)$ and those who resided in the capital city $(0.62,95 \% \mathrm{Cl} 0.47-0.83)$. Health professionals gave 'advice to exercise' most to patients with or at highest risk for diabetes, and these were more likely to engage in physical activity than those without diabetes receiving the same advice, net of the effect of other covariates ( $\mathrm{R}=3.68$ and 1.17 , respectively).

Conclusions: The inverse associations between physical activity and SES indicators suggest a negative influence of urbanization on activity levels of Lebanese adults. The missed opportunity for clinical primary preventive services for the majority non-diabetic population calls for population-based public health approaches that promote physical activity as a routine lifestyle in the general population.
\end{abstract}

Keywords: Type 2 diabetes mellitus, Lebanon, Physical activity

\footnotetext{
* Correspondence: am00@aub.edu.lb

'Department of Epidemiology and Population Health, Faculty of Health

Sciences, American University of Beirut (AUB), PO Box: 11-0236, Riad el Solh,

Beirut 1107-2020, Beirut-Lebanon

${ }^{6}$ Member of the Public Health and Nutrition (PHAN) Research Group at the

American, University of Beirut, Beirut, Lebanon

Full list of author information is available at the end of the article
} 


\section{Background}

The overall health benefits of physical activity have long been established. In 1953, Morris and colleagues showed that bus conductors in London experienced half the coronary heart disease mortality rates of their driver counterparts who spent their working days sitting behind the wheel [1]. Physical activity (PA) diminishes the risk of cardiovascular risk factors, and its role, notably in the prevention and management of type 2 diabetes, is well documented [2,3]. Today, lack of physical activity is the fourth leading risk factor for global mortality [4] and is one of the leading public health indicators [5]. Despite the known hazards of being sedentary, physical activity levels continue to fall worldwide [4]. In one article of the Lancet series dedicated to physical activity, Hallal and colleagues [6] note that roughly three out of ten adults aged 15 years and over are below the recommended physical activity threshold, and urge governments and populations to take physical inactivity as serious public health issue. The Middle East and North Africa (MENA) region, as a whole, is burdened with high prevalence rates of physical inactivity [6] reaching 82.3\% among Syrian adults [7] and 96.1\% in Saudi Arabia [8]. In parallel, the region has the highest comparative prevalence of diabetes (4\%-21\%) [9]. According to the International Diabetes Federation [10], six out of the world's top ten countries for highest prevalence of diabetes are from the MENA region.

Lebanon, a small-middle income country of the MENA region, is characterized by high urbanization rate (87\%), high literacy rate (89\%), and life expectancy approaching 73 years for men and 76 years for women. With westernization and socioeconomic changes, noncommunicable diseases have long emerged as the leading cause of morbidity and mortality [11]. Obesity rates have increased from $17.4 \%$ in 1997 to $28.2 \%$ in 2009 among adults aged 20 years and older [12] and close to one third of the adult population suffer from metabolic syndrome [13]. Yet, national data on the distribution of physical activity and diabetes are limited to a few small-scale surveys and remain deficient. Also, physical activity is influenced by a diverse range of factors, and the influence of these factors on the pattern of physical activity in the general population and among those with diabetes has not been studied. Using data from a recent nationally representative cross-sectional survey, this study intended to address these issues in order to inform public health policies and plans. The study aimed to answer the following questions: firstly, what is the prevalence of physical activity among the total population, and among those with diabetes and risk of developing diabetes; and secondly, what are the factors associated with the level of physical activity performed as stratified by diabetes status?

\section{Methods}

\section{Study design and participants}

The data used in this study were drawn from the national Nutrition and Non-Communicable Disease Risk Factor (NNCD-RF) survey conducted in Lebanon between 2008 and 2009 on a representative sample of Lebanese adults aged 25 and over of both sexes. This was a populationbased cross-sectional survey that followed the WHO STEPwise guidelines. The study sample was drawn based on random area probability multi-stage cluster sampling: the strata were the Lebanese Governorates, the clusters were selected at the level of districts, urban and rural, and the housing units constituted the primary sampling units in the different districts. Only one adult from each household was selected, excluding pregnant and lactating women and individuals with mental disabilities. Refusals at the household level did not exceed $10.7 \%$, yielding a sample size of 2195 adults aged 25 years and over. The study sample had enough power to detect an overall diabetes prevalence of $10 \%$, with an alpha of $5 \%$ and an error margin of $\pm 1.5 \%$. The protocol of the NNCD-RF was approved by the Institutional Review Board of the American University of Beirut and all subjects gave informed consent for their participation.

\section{Measures \\ Physical activity}

Physical activity, our main outcome measure, was assessed using the short version of the International Physical Activity Questionnaire (IPAQ) [14]. While the use of devices such as accelerometers and pedometers may provide a more reliable measure of $\mathrm{PA}$, however the IPAQ has been shown to be a reliable and valid tool to obtain comparable estimates of physical activity, based on activity undertaken across multiple domains including leisure, domestic, work and transport $[15,16]$. The questionnaire asks about three specific levels of activity: walking, moderate- and vigorous-intensity activities, and their frequency (days per week) and duration (minutes per day). For this study, a measure that describes the total volume of physical activity was calculated by weighting each type of activity by its energy requirements defined in METs (METs are multiples of the resting metabolic rate estimated at 3.3 for walking, 4.0 for moderate physical activity and 8.0 for vigorous physical activity) to yield a combined score in MET.minutes (computed by multiplying the MET score by the minutes performed within a week). As regular participation is a key concept in current public health guidelines for physical activity, both the total volume and the number of days/sessions are included in the IPAQ analysis algorithms, thus classifying our subjects into three categories: inactive, moderately active, and HEPA (Health Enhancing Physical Activity) [17]. The moderate level corresponded to 
meeting physical activity guidelines of 30 minutes of moderate intensity activity 5 days a week or 20 minutes of vigorous activity 3 days a week, or a combination of both. HEPA corresponded to either having vigorousintensity activity on $>3$ days/week while accumulating at least 1500 MET-minutes/week; or $>5$ days of any combination of walking, moderate-intensity, or vigorous-intensity intensity activities achieving at least 3000 MET-minutes per week. Subjects not meeting either criterion were classified as having low level of physical activity.

\section{Adults with diabetes and those at risk of developing diabetes}

Respondents were defined as 'having diabetes' based on self-reports to the question whether they had ever been diagnosed with diabetes by a health professional, excluding gestational diabetes. Using the American Diabetes Association criteria [18], we also defined individuals at 'risk of developing diabetes' as those non-diabetic subjects having one or more of the following six conditions: age over 45 years, overweight (BMI $\geq 25 \mathrm{~kg} / \mathrm{m}^{2}$ ), a diagnosis of hypertension, hyperlipidemia, cardiovascular disease and having family history of diabetes in first degree relatives. BMI was based on objective measures of height and weight, using calibrated methods and equipment. Whereas the presence of hyperlipidemia, cardiovascular disease and family history of diabetes were based on self-reports, blood pressure was objectively measured using a standardized mercury sphygmomanometer after participants were seated and rested for 5 minutes. The average of three measurements was considered and hypertension was judged to be present if the participant had a mean value for systolic/diastolic blood pressure $\geq 140 / 90 \mathrm{mmHg}$ and/ or was on medications for hypertension.

\section{Other covariates}

In addition to the above, other covariates were examined as factors associated with physical activity and these included gender, governorate (Beirut, the capital city vs. others), and education (low, middle, high). Low educational level included those with education less than primary schooling (equivalent to eight years of schooling), middle educational level included those who attended at least secondary or technical schooling (equivalent to 12 years of schooling), while those with a high educational level were holders of a university bachelor's degree or higher. We also included in our list of covariates 'having received health professional advice to exercise more' within the past six months prior to the survey. The number of cases with missing data was not substantial in the independent and dependant variables $(n=43$ in either variable), and this was dealt with by employing list-wise deletion.

\section{Data analysis}

Descriptive statistics, expressed as percentages and number of cases, were calculated for the total study population as well as across categories of physical activity levels. Prevalence estimates of physical activity and their 95\% confidence intervals (CI) were calculated for diabetic patients and those without diabetes according to their level of risk of developing diabetes. Evidence-based guidelines for physical activity among diabetic subjects indicate the need to be engaged in moderate to vigorous physical activity for at least 3 days a week [19]. Hence, physical activity was further grouped, for the logistic regression analysis, into a binary variable: physically active to include individuals with moderate and high levels of physical activity and the physically inactive. The regression analysis estimated the odds of being physically active among adults in the total sample, and among subjects with and without diabetes, separately. Covariates included in the models were age, gender, governorate, education, car ownership, smoking, BMI, hypertension and hyperlipidemia, heart disease, as well as receiving advice to exercise. Prevalence odds ratios (ORs) and their 95\% CIs were estimated. Statistical significance was defined as $p$-value $<0.05$, and SPSS 18.0 and STATA 10.0 software packages were used for analyses.

\section{Results}

The mean age of the study sample was 44.7 years $(\mathrm{SD} \pm 14.9)$ with a slightly larger proportion of females than males (53.6\% vs. $46.4 \%$, respectively) (Table 1$)$. The majority of participants had attained middle or high level education (75.4\%), resided outside the capital city of Beirut (89.2\%), and owned at least one car (71.5\%). Close to $38 \%$ were current smokers. Their mean BMI was $27.8 \mathrm{~kg} / \mathrm{m}^{2}$ $(\mathrm{SD}=5.4)$, with around $29 \%$ being obese $\left(\mathrm{BMI} \geq 30 \mathrm{Kg} / \mathrm{m}^{2}\right)$. Hypertension was the most prevalent health condition (31.8\%). This was followed by hyperlipidemia (18.5\%) and heart disease (8.0\%). Around one third of individuals were advised to exercise in the six months prior to the survey.

Table 1 also presents physical activity rates according to the various socio-demographic and health characteristics of the study sample (row \%s). Close to half of the survey participants reported low or no physical activity (46.7\%), 31.1\% reported moderate levels, while $22.2 \%$ reported HEPA levels. Physical activity, notably HEPA, varied with age being lowest among older adults aged 65 and above. Compared to their counterparts, physical activity was slightly higher among females, those living outside Beirut, and among participants with low-tomiddle educational levels. Obese respondents and those suffering from co-morbid conditions including hypertension, hyperlipidemia and heart disease were less likely to be performing HEPA. Respondents receiving 
Table 1 Baseline characteristics of study participants and rates of physical activity, Lebanon, 2009

\begin{tabular}{|c|c|c|c|c|c|c|c|c|}
\hline & \multirow{2}{*}{\multicolumn{2}{|c|}{$\begin{array}{c}\text { Baseline } \\
\text { distribution }\end{array}$}} & \multicolumn{6}{|c|}{ Rates of physical activity } \\
\hline & & & \multicolumn{2}{|c|}{ Inactive } & \multicolumn{2}{|c|}{ Moderate } & \multicolumn{2}{|c|}{ HEPA* } \\
\hline & $\mathrm{N}$ & $\%$ & $n$ & $\%$ & $\mathrm{n}$ & $\%$ & $\mathbf{n}$ & $\%$ \\
\hline Total Sample & 2195 & & 1026 & 46.7 & 682 & 31.1 & 487 & 22.2 \\
\hline \multicolumn{9}{|c|}{ Socio-demographic } \\
\hline \multicolumn{9}{|l|}{ Age } \\
\hline $25-44$ & 1245 & 56.7 & 608 & 48.8 & 348 & 28.0 & 289 & 23.2 \\
\hline $45-64$ & 664 & 30.3 & 271 & 40.8 & 239 & 36.0 & 154 & 23.2 \\
\hline$\geq 65$ & 286 & 13.0 & 147 & 51.4 & 95 & 33.2 & 44 & 15.4 \\
\hline \multicolumn{9}{|l|}{ Gender } \\
\hline Male & 1019 & 46.4 & 520 & 51.0 & 286 & 28.1 & 213 & 20.9 \\
\hline Female & 1176 & 53.6 & 506 & 43.0 & 396 & 33.7 & 274 & 23.3 \\
\hline \multicolumn{9}{|l|}{ Governorate } \\
\hline Beirut & 236 & 10.8 & 139 & 58.9 & 64 & 27.1 & 33 & 14.0 \\
\hline Other & 1959 & 89.2 & 887 & 45.3 & 618 & 31.5 & 454 & 23.2 \\
\hline \multicolumn{9}{|l|}{ Education } \\
\hline Low & 540 & 24.6 & 237 & 43.9 & 184 & 34.1 & 119 & 22.0 \\
\hline Middle & 952 & 43.4 & 418 & 43.9 & 300 & 31.5 & 234 & 24.6 \\
\hline High & 703 & 32.0 & 371 & 52.8 & 198 & 28.2 & 134 & 19.1 \\
\hline \multicolumn{9}{|l|}{ Owns a car } \\
\hline None & 547 & 24.9 & 215 & 39.3 & 197 & 36.0 & 135 & 24.7 \\
\hline 1 or more & 1648 & 71.5 & 811 & 49.2 & 485 & 29.4 & 352 & 21.4 \\
\hline
\end{tabular}

Health-related

\begin{tabular}{|c|c|c|c|c|c|c|c|c|}
\hline \multicolumn{9}{|l|}{ Smoking } \\
\hline Never & 1217 & 55.4 & 557 & 45.8 & 401 & 32.9 & 259 & 21.3 \\
\hline Past & 150 & 6.8 & 78 & 52.0 & 41 & 27.3 & 31 & 20.7 \\
\hline Current & 828 & 37.7 & 391 & 47.2 & 240 & 29.0 & 197 & 23.8 \\
\hline \multicolumn{9}{|l|}{ BMI } \\
\hline Normal & 719 & 32.8 & 336 & 46.7 & 216 & 30.0 & 167 & 23.2 \\
\hline Overweight & 832 & 37.9 & 359 & 43.1 & 275 & 33.1 & 198 & 23.8 \\
\hline Obese & 638 & 29.1 & 328 & 51.4 & 190 & 29.8 & 120 & 18.8 \\
\hline \multicolumn{9}{|l|}{ Hypertension } \\
\hline No & 1489 & 67.8 & 698 & 46.9 & 447 & 30.0 & 344 & 23.1 \\
\hline Yes & 699 & 31.8 & 324 & 46.4 & 234 & 33.5 & 141 & 20.2 \\
\hline \multicolumn{9}{|l|}{ Hyperlipidemia } \\
\hline No & 1790 & 81.5 & 815 & 45.5 & 553 & 30.9 & 422 & 23.6 \\
\hline Yes & 405 & 18.5 & 211 & 52.1 & 129 & 31.9 & 65 & 16.0 \\
\hline \multicolumn{9}{|l|}{ Heart disease } \\
\hline No & 2019 & 92.0 & 926 & 45.9 & 628 & 31.1 & 465 & 23.0 \\
\hline Yes & 176 & 8.0 & 100 & 56.8 & 54 & 30.7 & 22 & 12.5 \\
\hline \multicolumn{9}{|c|}{ Advice to exercise } \\
\hline No & 1544 & 70.3 & 724 & 46.9 & 454 & 29.4 & 366 & 23.7 \\
\hline Yes & 651 & 29.7 & 302 & 46.4 & 228 & 35.0 & 121 & 18.6 \\
\hline
\end{tabular}

*HEPA, health enhancing physical activity.

Some totals do not add up to 2195 or $100 \%$ due to missing data. medical advice to exercise were more likely to engage in moderate levels of physical activity.

Survey participants with diagnosed diabetes made up $8.5 \%$ of the study sample (Table 2). An additional 26.3\% and $4.1 \%$ of the subjects without diabetes had 3-4 and 5-6 diabetes risk factors, respectively. HEPA prevalence rates decreased consistently with increasing number of diabetes risk factors ( $p$-value $<0.001$ ) and was significantly lower among those with diabetes than those without diabetes ( $9.6 \%$ vs. $23.4 \%$, p-value $<0.001)$.

Results of the multivariable logistic regression model for the total sample and stratified by diabetes status are shown in Table 3. In the total sample, physical activity was significantly higher among the middle-age group (45-64 years) (OR=1.46, 95\% CI 1.17-1.81), females $(\mathrm{OR}=1.30$, 95\% CI 1.09-1.55), and among respondents residing in governorates outside the capital city of Beirut $(\mathrm{OR}=1.61,95 \%$ CI 1.21-2.62). In contrast, participants with high levels of education (OR=0.75, 95\% CI 0.57 $0.98)$ and those who owned at least one car $(\mathrm{OR}=0.71$, 95\% CI $0.57-0.88$ ) were significantly less likely to be physically active. Whilst hypertension did not associate with physical activity, obesity (OR=0.78, 95\% CI 0.62 0.99 ) and pre-existing health conditions significantly decreased the odds of physical activity $(\mathrm{OR}=0.66,95 \% \mathrm{CI}$ $0.51-0.85$ for hyperlipidemia; OR $=0.61,95 \%$ CI $0.43-$ 0.87 for heart disease). A significant association was also noted for receiving health professional advice to engage in physical activity (OR=1.25, 95\% CI 1.01-1.54).

Findings of the regression analysis varied according to diabetes status. Owing to the relatively large proportion of subjects without diabetes in our study sample (91\%), the direction and strength of the associations among the non-diabetic subjects were, overall, similar to those estimated for the total sample. Among diabetic subjects, we note that the results were, in the majority, not significant and with wide confidence intervals. However, the association of physical activity with heart disease $(\mathrm{OR}=0.29$, 95\% CI 0.13-0.70) and with receiving professional advice to exercise were further enhanced among subjects with diabetes (OR=3.68, 95\% CI 1.55-8.74), compared to those without diabetes.

\section{Discussion}

Findings of this study indicate that close to one third (31\%) of Lebanese adults, 25 years of age and older, participate in moderate levels of physical activity and only $22 \%$ engage in health enhancing energy expenditure levels. These results are lower than those reported in the International Prevalence Study conducted in 20 countries worldwide using the same instrument as ours (median 33\% and 40\% for moderate and vigorous activity, respectively) [20]. Compared to their counterparts, physical activity was lower in subgroups with urban 
Table 2 Rates of physical activity among Lebanese adults diagnosed with diabetes and at risk for developing diabetes, 2009

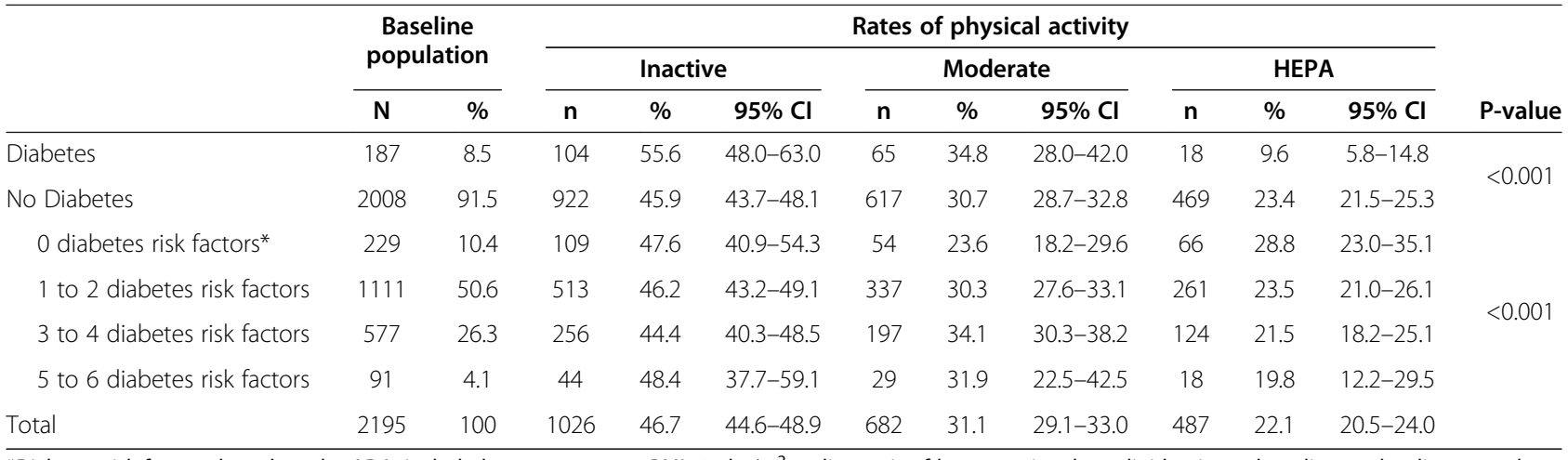

*Diabetes risk factors, based on the ADA, included: age $\geq 45$ years, $\mathrm{BMl} \geq 25 \mathrm{~kg} / \mathrm{m}^{2}$, a diagnosis of hypertension, hyperlipidemia, and cardiovascular disease and family history of diabetes.

characteristics and lifestyle, namely those with high levels of education, who owned at least one car, and among those who resided in Beirut, the capital city. Given the clear evidence in the literature of the health benefits of physical activity in the prevention and management of diabetes, it is disturbing to note that HEPA rates decreased consistently with increasing number of diabetes risk factors and were only $10 \%$ among subjects known to have diabetes.

During the last few years, different subgroups of the Lebanese adult population have been studied with regard to physical activity with varying results. For example, Sibai and colleagues found approximately 16\% of HEPA levels in a sample of 499 adult population attending health centers in Lebanon [13]. Tohme and colleagues [21] estimated the prevalence rate of daily exercise for 30 minutes in a nationally representative sample of 2,125 subjects at $20.4 \%$. A cross sectional study conducted among 346 adults 18 years and over selected from large stores across all governorates yielded a much higher estimate (55\%) [22]. Findings from the WHO STEPwise surveys in countries of the MENA region also show a wide range of prevalence estimates of physical activity, varying between 13.2\% and $78.4 \%$ [23]. The outcomes of these studies are difficult to compare with findings from the present study owing to differences in sampling design, sample characteristics, as well as the tool, operational definition and the scoring method employed to measure physical activity.

We found inverse associations between physical activity and various indicators of socio-economic status (SES), as illustrated by education $(\mathrm{OR}=0.75)$, car ownership $(\mathrm{OR}=0.71)$, and residence in Beirut, the highly urbanized metropolitan capital city of the country $(\mathrm{OR}=0.62)$. Reviews of SES correlates with physical activity show mostly positive associations; this is particularly evident for education $[24,25]$. Yet, this evidence has largely been noted for leisure-time physical activity with diverse and often crude measures being employed $[6,24]$.
In comparison, our assessment of physical activity was based on the IPAQ that includes energy expenditure from several domains, and captures expenditure from lifestyle activities integrated in daily routines of work, household chores and transportation, additional to leisure time activities. Thus, higher education, car ownership and residence inside the capital city can mean more sedentary jobs, reduced walking and less physically active lifestyles. Support for our results is found in studies from subSaharan Africa where urban dwellers had a significantly lower physical activity energy expenditure than rural dwellers $(44.2 \pm 21.0$ vs. $59.6 \pm 23.7 \mathrm{~kJ})$ [26], and in selected developing countries of the Asian-Pacific and the MENA regions [14,27]. Similarly, cross-country comparisons at the ecological level show that physical activity is more common in low income countries than in high income countries $[6,28]$.

Consistent with findings from the literature [3], the presence of obesity and co-morbidities associated inversely with physical activity among our study participants [3]. Also, HEPA levels declined consistently as the number of diabetes risk factors increased, and was lowest among people with diabetes. Given the benefits of regular moderate physical exercise as a form of intervention management for improving $\mathrm{HbA1c}$ levels and cardiovascular risk factors in diabetic patients, it is concerning that the majority of Lebanese adults with diabetes remain either sedentary or insufficiently active (55.6\%) and only $9.6 \%$ participate in health enhancing activities. Our results are similar to those found among 406 consecutive patients attending the Diabetes clinic in Dundee, UK (9\%) [29], yet much lower than findings from other patient groups in the National Health and Nutrition Examination Survey (28.2\%) and in the Medical Expenditure Panel Survey (39\%) in the US [30,31].

Patients receiving advice from a health professional to alter their lifestyle and exercise were found to be significantly more likely to engage in physical activity than non- 
Table 3 Correlates of physical activity in the total sample and stratified by diabetes status among Lebanese adults: results of the logistic regression, 2009

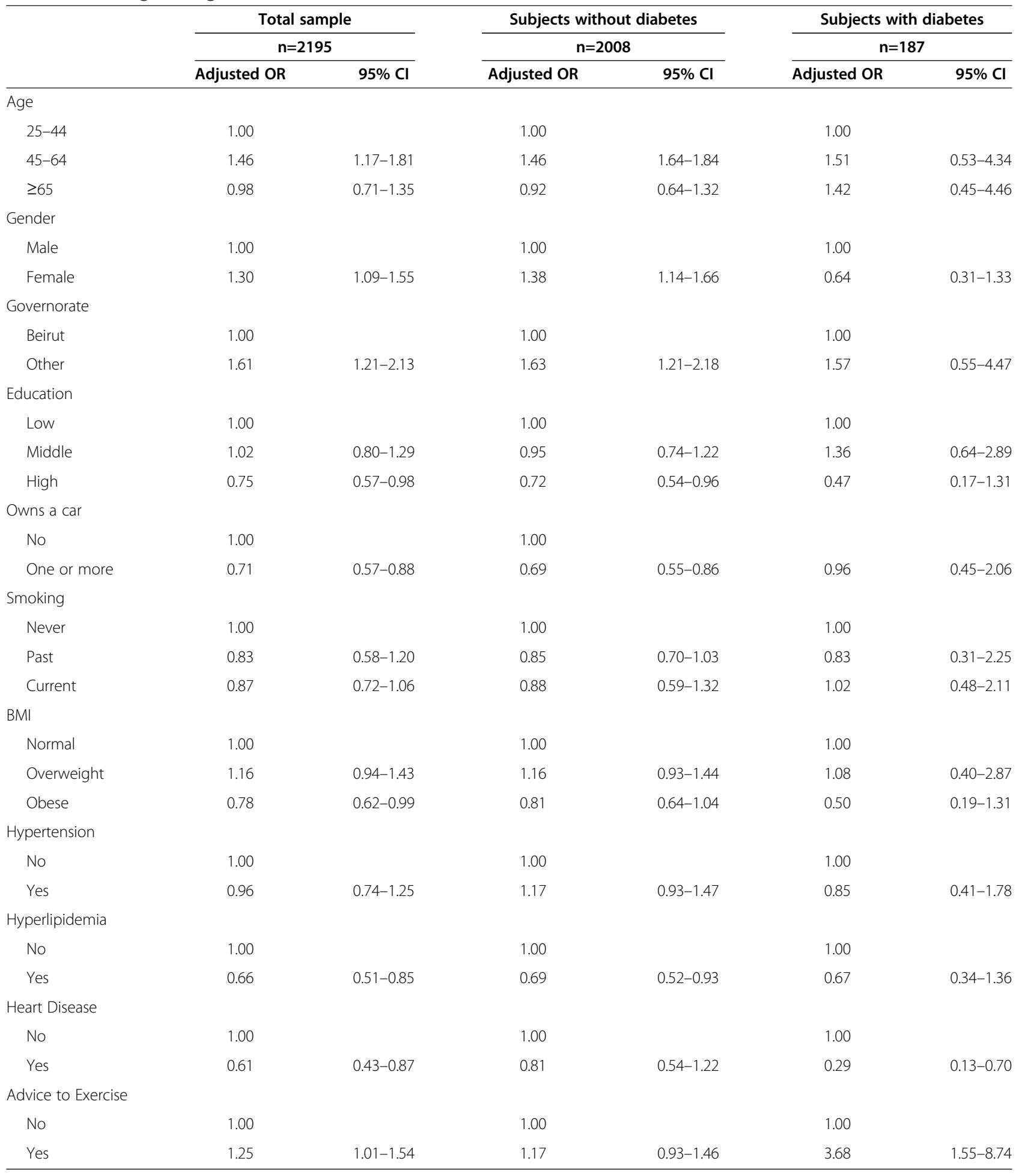

diabetic subjects receiving the same advice, net of the effect of other covariates $(\mathrm{OR}=3.68$ and $\mathrm{OR}=1.17$, respectively). This finding merits further consideration. Post hoc analysis of our data shows a positive gradient between receiving advice and the presence of diabetes risk factors (p-value for trend $<0.01$ ) with health professionals advising most patients with or at highest risk for diabetes $(76.5 \%$ and $71.4 \%$, respectively) and least those without diabetes 
(13.1\%). While this implies the recognition by health professionals of the importance of physical activity among diabetes patients on one end of the spectrum, it also suggests a missed opportunity for primary disease prevention in the clinic for the majority non-diabetic population, on the other end. Given the crucial role of physical activity in the prevention and management of diabetes, these results have important implications to health care providers and policy makers with regard to diabetes control guidelines and protocols. Whereas studies are needed to examine contextual factors that influence behavioral change of patients and non-patients in Lebanon, it is the time to start targeting interventions that promote physical activity in the general as a life-style to be adopted. The challenge remains to alert physicians towards primary disease prevention services for the non-diabetic majority and to re-direct policy attention to diabetes control from the high-risk emphasis towards the population-based public health approach.

The findings of this study are considered in light of the following limitations. Because of the cross-sectional nature of the study, the temporality of certain associations cannot be established with confidence. Also, the study lacked sufficient power to detect significant correlates of physical activity among subjects with diabetes $(n=187)$. Whereas BMI and hypertension were objectively measured using calibrated equipment and standardized techniques, data on diabetes status are self-reported, introducing possible misclassification bias. However, the amount of bias is minimal as post-hoc analysis of our data showed that those who reported having diabetes were mostly on medication and/or were following a diet (84\%). Also, diabetes is an overt disease, and so diabetic individuals are likely to be referred to a physician for diagnosis and follow-up. Furthermore, studies from Lebanon and elsewhere show substantial agreement between self-report and medical record diagnosis for several medical conditions including diabetes (kappa range 0.71-0.78) and heart disease (kappa range 0.83-0.87) [32-34]. Although the IPAQ's validity has originally been assessed for individuals aged up to 69 years of age, nonetheless, studies in the developed world and in the region have used and validated it on samples aged over 70 years $[35,36]$.

In spite of the above caveats, this is the first study that we are aware of to assess the prevalence of physical activity and its correlates with a focus on diabetes and diabetes risk factors in a large national sample in Lebanon and the MENA region. Estimates of physical activity from national surveys such as the current one can provide valuable information to guide national policies and serve as a benchmark for monitoring and evaluation thereafter. Furthermore, our assessment of physical activity based on the IPAQ captures more contributions towards total physical activity levels and does not ignore routine activities undertaken by housewives, for example, who constitute a good percent of the Lebanese female population $(67.5 \%)$. The ability to quantify benefit from several domains including routine daily pursuits, whether at work or home, may be more applicable to health promotion initiatives in low-income countries such as Lebanon where access to leisure-time activity is hampered by the cost of private club memberships and the lack of public and age-friendly outdoor spaces to exercise.

\section{Conclusions}

In conclusion, population-based approaches for control and prevention of diabetes by physical activity should be innovative while addressing the patient's concepts and community perspectives of health and illness and understanding the complex interaction between barriers and facilitators across the different levels of the individual, family, community and society. Structural factors may limit our population's engagement of physical activity [37], hence the need for governmental policies that are appropriate to population's context. Yet the statement made by the National Board of Health and Welfare in Sweden as early as the 1970s to "take every opportunity to increase life style activities" remains the most forceful and affordable message to be adopted by policymakers at the population-level in developing countries.

\section{Abbreviations}

BMI: Body mass index; HEPA: Health enhancing physical activity; IPAQ: International physical activity questionnaire; MENA: Middle east and North Africa; MET: Metabolic equivalent; NNCD-RF: Nutritional and Noncommunicable disease risk factor.

\section{Competing interests}

The authors declare that they have no competing interests.

\section{Authors' contributions}

AMS contributed towards study design, study logistics, data collection, data analysis, and interpretation and manuscript drafting. RT contributed toward study logistics, data collection, analysis and interpretation. SA contributed to conception of the hypothesis, the analysis and write-up of the paper. $\mathrm{NH}$ contributed to study logistics, data collection and data interpretation. CC contributed to the conception of the hypothesis, analysis and write-up. All authors provided critical insight, and revisions to the manuscript. All authors read and approved the final version of the manuscript submitted for publication.

\section{Acknowledgements}

The writing up of this paper was partly funded by the RESCAP-MED fellowship program: European Commission FP7 funding (2012-2014) grant no: 281640 .

\section{Funding}

The study was funded by the Training Programs in Epidemiology and Public Health Interventions Network (TEPHINET-CDC), the World Health Organization (WHO)- Lebanon, and the Lebanese National Council for Scientific Research.

\section{Author details}

${ }^{1}$ Department of Epidemiology and Population Health, Faculty of Health Sciences, American University of Beirut (AUB), PO Box: 11-0236, Riad el Solh, Beirut 1107-2020, Beirut-Lebanon. ${ }^{2}$ Affiliation at the time of the study: Department of Epidemiology and Population Health, Faculty of Health 
Sciences, American University of Beirut, Beirut, Lebanon. ${ }^{3}$ Current affiliation: Centers for Disease Control and Prevention, Atlanta, GA 30333, USA ${ }^{4}$ Lebanese University, Faculty of Medical Sciences, Beirut, Lebanon. ${ }^{5}$ Department of Nutrition and Food Science, Faculty of Agricultural and Food Sciences, American University of Beirut, Beirut, Lebanon. ${ }^{6}$ Member of the Public Health and Nutrition (PHAN) Research Group at the American, University of Beirut, Beirut, Lebanon.

Received: 24 April 2013 Accepted: 11 October 2013 Published: 24 October 2013

\section{References}

1. Morris JN, Heady JA, Raffle PA, Roberts CG, Parks JW: Coronary heartdisease and physical activity of work. Lancet 1953, 265:1053-1057.

2 Lee IM, Shiroma EJ, Lobelo F, Puska P. Blair SN, Katzamarzyk PT: Effect of physical inactivity on major non-communicable diseases worldwide: an analysis of burden of disease and life expectancy. Lancet 2012, 380:219-229.

3. Luke A, Dugas LR, Durazo-Arvizu RA, Cao G, Cooper RA: Assessing physical activity and its relationship to cardiovascular risk factors: NHANES 20032006. BMC Public Health 2011, 11:387.

4. Kohl HW 3rd, Craig CL, Lambert EV, et al: The pandemic of physical inactivity: global action for public health. Lancet 2012, 380:294-305.

5. Centers for Disease Control and Prevention: Surgeon General's Report on Physical Activity and Health. Springfield, VA: National Technical Information Service; 1996.

6. Hallal PC, Anderson LB, Bull FC, et al: Global physical activity levels: surveillance progress, pitfalls, and prospects. Lancet 2012, 380:247-257.

7. Al Ali R, Rastam S, Fouad MF, Mzayek F, Maziak W: Modifiable cardiovascular risk factors among adults in Aleppo, Syria. Int J Public Health 2011, 56:653-662.

8. Mabry RM, Reeves MM, Eakin EG, Owen N: Evidence of physical activity participation among men and women in the countries of the gulf cooperation council: a review. Obes Rev 2010, 11(6):457-464.

9. Badran M, Laher I: Type II diabetes mellitus in Arabic-speaking countries. Int J Endo 2012. doi:10.1155/2012/902873.

10. International Diabetes Foundation: Rates of diabetes in the Middle East to double in next 20 years. 2011. Accessed on August 1st 2012: http:/www.idf. org/sites/default/files/attachments/MENA-Press-Release-WDD.pdf.

11. Sibai AM, Fletcher A, Hills M, Campbell O: Non-communicable disease mortality rates using the verbal autopsy in a cohort of middle-aged and older populations in Beirut during wartime, 1983-93. J Epidemiol Community Health 2001, 55:271-276.

12. Nasreddine L, Naja F, Chamieh MC, Adra N, Sibai AM, Hwalla N: Trends in overweight and obesity in Lebanon: evidence from two national crosssectional surveys (1997 and 2009). BMC Public Health 2012, 12(1):798 doi:10.1186/1471-2458-12-798.

13. Sibai AM, Obeid O, Batal M, Adra N, El Khoury D, Hwalla N: Prevalence and correlates of metabolic syndrome in an adult Lebanese population. CVD Prev Control 2008, 3:83-90.

14. Bauman A, Cuevas F, Omar Z, et al: Cross-national comparison of socioeconomic differences in the prevalence of leisure-time and occupational physical activity, and active commuting in six Asia-Pacific countries. J Epidemiol Community Health 2011, 65:35-4312.

15. Hagströmer M, Oja P, Sjöström M: The international physical activity questionnaire (IPAQ): a study of concurrent and construct validity. Public Health Nutr 2005, 9:755-762.

16. Papathanasiou G, Georgoudis G, Papandreou M, et al: Reliability measures of the short international physical activity questionnaire (IPAQ) in Greek young adults. Hellenic J Cardiol 2009, 50:283-294.

17. Guidelines for data processing and analysis of the international physical activity questionnaire (IPAQ)- short and long forms. 2005. Accessed at http://www.ipaq.ki.se/scoring.pdf.

18. American Diabetes Association: Screening for type 2 diabetes. Diabetes Care 2003, 26:521-s24.

19. American Diabetes Association and National Institute of Diabetes, Digestive \& Kidney Diseases: The prevention or delay of type 2 diabetes. Diabetes Care 2002, 25:742-749

20. Bauman A, Bull F, Chey $T$, et al: The international prevalence study on physical activity: results from 20 countries. Int I Behav Nutr Phy 2009, 6:21.

21. Tohme RA, Jurjus AR, Estephan A: The prevalence of hypertension and its association with other cardiovascular disease risk factors in a representative sample of the Lebanese population. J Hum Hypertens 2005, 19:861-868.

22. Al-Tannir M, Kobrosly S, Itani T, El-Rajab M, Tannir S: Prevalence of physical activity among Lebanese adults: a cross-sectional study. J Phys Act Health 2009, 6:315-320

23. Sibai AM, Nasreddine L, Mokdad HA, Adra N, Tabet M, Hwalla N: Nutrition transition and cardiovascular disease risk factors in middle east and north Africa countries: reviewing the evidence. Ann Nutr Metab 2010, 57:193-203

24. Trost SG, Owen N, Bauman AE, Sallis JF, Brown W: Correlates of adults' participation in physical activity: review and update. Med Sci Sports Exerc 2002, 34:1996-2001

25. Gidlow C, Johnston LH, Crone D, Ellis N, James D: A systematic review of the relationship between socio-economic position and physical activity. Health Educ J 2006, 65:338-367.

26. Assah FK, Ekelund U, Brage S, Mbanya JC, Wareham NJ: Urbanization, physical activity, and metabolic health in Sub-Saharan Africa. Diabetes Care 2011, 34:491-496.

27. Merom D, Sinnreich R, Aboudi V, Kark JD, Nassar H: Lifestyle physical activity among urban Palestinians and Israelis: a cross-sectional comparison in the Palestinian-Israeli Jerusalem risk factor study. BMC Public Health 2012, 12:90

28. Dumith SC, Hallal PC, Reis RS, Kohl HW 3rd: Worldwide prevalence of physical inactivity and its association with human development index in 76 countries. Prev Med 2011, 53:24-28.

29. Thomas N, Alder E, Leese GP: Barriers to physical activity in patients with diabetes. Postgrad Med J 2004, 80:287-291.

30. Resnick HE, Foster GL, Bardsley J, Ratner RE: Achievement of American diabetes association clinical practice recommendations among U.S. Adults with diabetes, 1999-2002: the national health and nutrition examination survey. Diabetes Care 2006, 26:531-537.

31. Morrato EH, Hill JO, Wyatt HR, Ghushchyan V, Sullivan PW: Physical activity in U.S. adults with diabetes and at risk for developing diabetes, 2003. Diabetes Care 2007, 30:203-209.

32. Halabi S, Zurayk H, Awaida R, Darwich M, Saab B: Reliability and validity of self and proxy reporting of morbidity data: a case study from Beirut, Lebanon. Int J Epidemiol 1992, 21:607-12.

33. Okura Y, Urban LH, Mahoney DW, Jacobsen SJ, Rodeheffer RJ: Agreement between self-report questionnaires and medical record data was substantial for diabetes, hypertension, myocardial infarction and stroke but not for heart failure. J Clin Epidemiol 2004, 57:1096-1103.

34. Tisnado DM, Adams JL, Liu H, et al: What is the concordance between the medical record and patient self-report as data sources for ambulatory care? Med Care 2006, 44:132-140.

35. Ainsworth BE, Macera CA, Jones DA, et al: Comparison of the 2001 BRFSS and the IPAQ physical activity questionnaires. Med Sci Sports Exer 2006 38:1584-1592

36. Al-Hazzaa HM: Heath-enhancing physical activity among Saudi adults using the international physical activity questionnaire (IPAQ). Public Health Nutr 2007, 10:59-64.

37. Sibai AM, Hwalla N, Adra N, Rahal M: Prevalence and covariates of obesity in Lebanon: findings from the first epidemiological study. Obes Res 2003, 11:1353-1361

doi:10.1186/1471-2458-13-1002

Cite this article as: Sibai et al:: Physical activity in adults with and without diabetes: from the 'high-risk' approach to the 'populationbased' approach of prevention. BMC Public Health 2013 13:1002. 